\title{
The Onsala blind 6.7 GHz survey of the galactic plane: new methanol masers in the northern hemisphere
}

\author{
Michele R. Pestalozzi, Vincent Minier, Roy Booth, and John Conway \\ Onsala Space Observatory, S-439 92 Onsala, Sweden
}

\begin{abstract}
We review the state of the Onsala blind survey of the galactic plane, searching for new $6.7 \mathrm{GHz}$ methanol masers. We also describe preliminary results of millimeter follow-up observations of the new detections and high resolution observations using the EVN. We conclude that blind surveys are important to complement targeted searches done until now and give the possibility to detect new classes of objects.
\end{abstract}

\section{Introduction}

Methanol masers are often detected in regions with typical signposts of massive star formation, such as $\mathrm{H}_{2} \mathrm{O}$ and $\mathrm{OH}$ masers, and some of them are detected towards IRAS sources satisfying the Wood \& Churchwell (1989) criterium for UCHII regions. Nevertheless high resolution VLBI observations show that such masers are not necessarily cospatial with IRAS UCHII regions (Minier, Conway, \& Booth 2001), which leads us to suppose that the maser activity can appear at a very early stage in the formation of a star, before the formation of a UCHII region. This fact increases the importance of blind surveys for $6.7 \mathrm{GHz}$ masers of the whole galactic plane.

We are using the Onsala $25 \mathrm{~m}$ telescope to conduct such a survey $(|b| \leq$ $0.55^{\circ}, 35^{\circ} \leq l \leq 220^{\circ}$ ), searching for the powerful $6.7 \mathrm{GHz}$ maser line of methanol. Since late 1998 we have surveyed about $45 \mathrm{deg}^{2}$, and have made 11 detections (see Table 1). Our unbiased survey should be regarded as complementary to the work carried out towards the Galactic center from Australia (Caswell 1996a+b, Ellingsen 1996).

The newly upgraded Onsala $25 \mathrm{~m}$ telescope is equipped with a three stage cryogenically cooled High Electron Mobility Transistor (HEMT) amplifier mounted at the secondary focus. The backend consists of a 1600 channel autocorrelator fed with a $6.4 \mathrm{MHz}$ bandwidth, which guarantees a complete coverage of the velocity distribution in the Galaxy $( \pm 150 \mathrm{~km} / \mathrm{s})$. Each square degree of the galactic plane is surveyed following an equilateral triangular grid, integrating 10 minutes per position. The system temperature lies between 1200 and $1500 \mathrm{Jy}$. Our sensitivity is about $6 \mathrm{Jy} /$ channel at $5 \sigma \mathrm{rms}$. The positions of 10 of the 11 detections were accurately determined using the $100 \mathrm{~m}$ Effelsberg telescope (September 2000, 10 arcsec accuracy) and those positions were used for the follow-up VLBI and millimeter observations. 


\section{Results from the blind survey}

Among the 11 detections, some masers have already been detected during other surveys; this allows us to test our system sensitivity. 5 detections have apparently been missed by previous pointed surveys. This allows us to make some conclusions about the selection criteria. For instance, most of the pointed surveys conducted until now made use of color filtering of the IRAS Point Source Catalogue (PSC). The reason for that is that class II methanol masers (such as that at $6.7 \mathrm{GHz}$ ) are supposed to appear in the neighbourhood of a strong infrared source which guarantees the pumping of the non thermal maser emission. A cross check of the detection rate in previous pointed surveys shows a relatively low efficiency in terms of number of detections of all those selection criteria: except for the surveys by Menten et al. (1991) and Caswell et al. (1995), the average detection rate is about $20 \%$ (see Table 2).

A comparison between IRAS-targeted and blind surveying in the regions observed at Onsala shows that, if we had applied a targeted search, we would have missed between 5 and 7 methanol maser sources, depending on the filtering applied to the IRAS catalogue (we considered three possiblities, Szymczak et al. 2000 , Wood \& Churchwell 1989, Schutte et al. 1993, ordered by decreasing number of IRAS sources included). This fact strongly justifies the worth of a blind survey.

Where no IRAS counterpart was found, we searched for Mid-IR sources around the methanol maser positions listed in the MSX catalogue (Midcourse Space Experiment). The fact is that for some sources not even a Mid-IR counterpart is found indicating that the methanol masers must arise in very compact and deeply embedded sources where only longer wavelengths $(\lambda>100 \mu \mathrm{m})$ can pass through the optically thick dust cocoon. Further observations at 450 and $850 \mu \mathrm{m}$ will be hopefully performed in order to clarify the origin of these sources.

\section{Follow-up observations}

The follow-up program of the Onsala survey consists of both a searching for and mapping of traditional high density tracers $\left({ }^{13} \mathrm{CO}, \mathrm{C}^{18} \mathrm{O}, \mathrm{CS}, \mathrm{HCO}^{+}, \mathrm{HCN}\right.$, thermal $\left.\mathrm{CH}_{3} \mathrm{OH}, \mathrm{HC}_{3} \mathrm{CN}\right)$ in the millimeter band $(85-110 \mathrm{GHz})$ using the Onsala $20 \mathrm{~m}$ telescope, and high resolution observations using the EVN. Because of the spectral simplicity of the new methanol masers (see e.g. Figure 1 and for comparison Minier et al. in this volume) it is difficult to make conclusions about the shape of the emitting region. The maps in the millimeter range suggest, on the other hand, that the methanol masers could be offset from the highest density gas, as shown in Figure 2.

\section{References}

Wood D.O.S., Churchwell E. 1989, ApJS, 69, 831

Caswell J.L. 1996a, MNRAS, 279, 79

Caswell J.L. 1996b, MNRAS, 283, 606

Ellingsen S.P. 1996, PhD Thesis, University of Tasmania

Menten K.M. 1991, ApJ, 380, 75 


\begin{tabular}{|c|c|c|c|c|c|c|}
\hline Source name & $\mathrm{RA}_{(J 2000)}$ & $\operatorname{Dec}_{(J 2000)}$ & $\mathrm{S}_{\text {peak }}$ & $v_{\text {peak }}$ & $\Delta v$ & Reference \\
\hline G 35.88-0.20* & $18: 57: 30.7$ & $02: 31: 45$ & 3.0 & 60 & 55,65 & new det \\
\hline G $40.25-0.19$ & $19: 05: 32.6$ & $06: 25: 38$ & 12 & 70 & 69,71 & 1 \\
\hline G 40.61-0.09 & 19:05:50.2 & $06: 47: 18$ & 4.5 & 27 & 26,28 & 2 \\
\hline G 41.34-0.14 & 19:07:22.1 & 07:25:12 & 51 & 14 & 13,16 & new det \\
\hline G $42.07+0.24$ & $19: 07: 20.8$ & 08:14:13 & 11 & 12 & 10,14 & new det \\
\hline G $43.17+0.01$ & 19:10:14.3 & 09:06:14 & 15 & 2 & $-2,16$ & 2 (W49) \\
\hline G $43.80-0.13$ & $19: 11: 54.3$ & $09: 35: 53$ & 30 & 37 & 34,41 & 2 \\
\hline G $44.05+0.00$ & 19:11:54.3 & 09:53:02 & 4.5 & 40 & 38,42 & new det \\
\hline G $45.07+0.13$ & $19: 13: 22.4$ & $10: 50: 58$ & 31 & 57 & 56,58 & 2 \\
\hline G $45.53+0.13$ & $19: 14: 14.8$ & $11: 15: 16$ & 5.5 & 63 & 53,65 & 3 \\
\hline G $85.40-0.07$ & $20: 54: 15.3$ & $44: 53: 54$ & 45 & -30 & $-32,-28$ & new det \\
\hline
\end{tabular}

Table 1. Source list of detected $6.7 \mathrm{GHz}$ methanol masers with coordinates in J2000, flux density (Jy), LSR velocity and velocity range of the strongest maser feature $(\mathrm{km} / \mathrm{s})$, as of May 2001. The source marked with a star has been detected in March 2001, its position comes from measurements at the $25 \mathrm{~m}$ telescope at Onsala (30 arcsec accuracy). In the last column references to previous observations are listed: 1) Szymczak et al. 2000, 2) Menten 1991, 3) Caswell et al. 1995.

\begin{tabular}{lll}
\hline Biased surveys & \\
\hline Menten (1991) & $80 / 123$ & UCHII, $\mathrm{H}_{2} \mathrm{O}, \mathrm{OH}$ \\
Schutte et al. (1993) & $35 / 235$ & IRAS, color selected \\
Caswell et al. (1995) & 245 & $\mathrm{OH}(208), \mathrm{SFR}$ \\
van der Walt et al. (1995) & $31 / 520$ & IRAS (UCHII) \\
Walsh et al. (1997) & $201 / 535$ & IRAS (color selected) \\
Slysh et al. (1999) & $42 / 429$ & IRAS (UCHII) \\
Szymczak et al. (2000) & $182 / 1399$ & IRAS (color selected) \\
$\rightarrow$ Detection rate towards IRAS sources: $\sim \mathbf{2 0} \%$
\end{tabular}

\begin{tabular}{lll}
\hline Blind surveys & & \\
\hline Caswell (1996a,b) & 80 masers in $11 \mathrm{deg}^{2}$ & $\rightarrow 7.2$ masers $/ \mathrm{deg}^{2}$ \\
Ellingsen $(1996)$ & 107 masers in $33 \mathrm{deg}^{2}$ & $\rightarrow 3.2$ masers $/ \mathrm{deg}^{2}$ \\
Onsala $(1999-2001)$ & 11 masers in $45 \mathrm{deg}^{2}$ & $\rightarrow \sim 0.25$ masers $/ \mathrm{deg}^{2}$ \\
\hline \hline
\end{tabular}

Table 2. Comparison between biased surveys and blind surveys of methanol. Notice the low efficiency of the IRAS color selected surveys in terms of detections. The particularly low detection rate of the Onsala survey can be the result of several factors: from Onsala we do not see the galactic center, fewer spiral arms are visible, slight lower sensitivity and possible variability of the sources. 


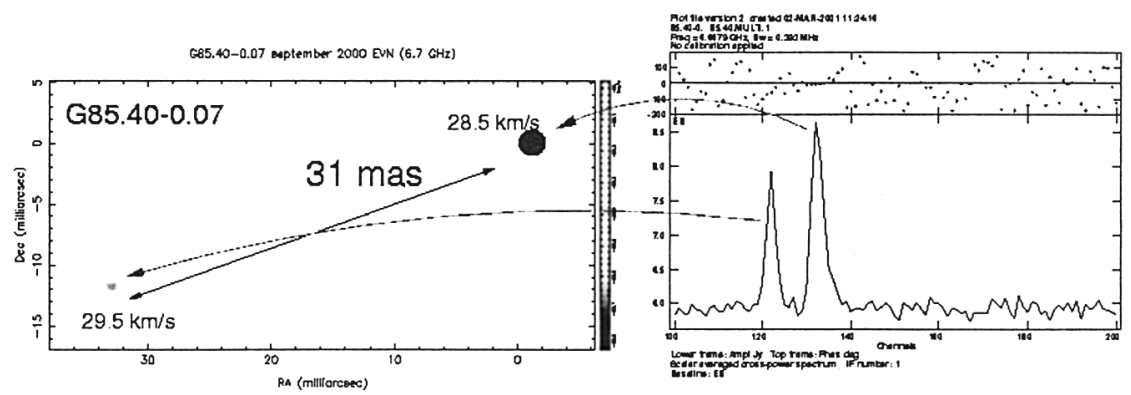

Figure 1. EVN cross-spectrum (baseline EB-TR) and relative position map of the source G 85.40-0.07. The simplicity of the spectrum does not allow any conlcusion about the shape of the emitting region. The distance between the two emission points is $180 \mathrm{AU}$ at the estimated kinematical heliocentric distance of $6 \mathrm{kpc}$. The velocity dispertion of the maser feature in the spectrum is $2 \mathrm{~km} / \mathrm{s}$.
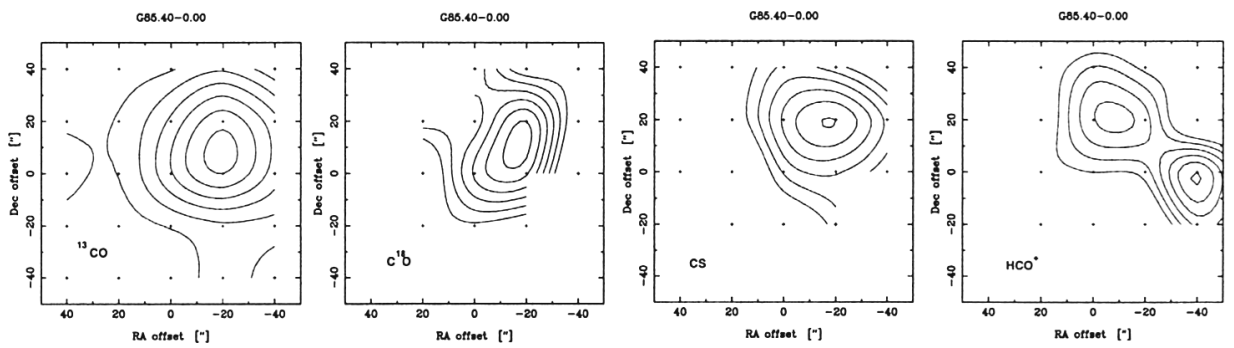

Figure 2. $m$ maps in ${ }^{13} \mathrm{CO}, \mathrm{C}^{18} \mathrm{O}, \mathrm{CS}$ and $\mathrm{HCO}^{+}$around the source $\mathrm{G} 85.40-0.07$. The first contour is at $50 \%$ of the peak emission, spacing $10 \%$. Map spacing is 20 arcsec. The methanol maser is located at the $(0,0)$ position. The position accuracies are 5 arcsec for the maps, 10 arcsec for the methanol maser.

Schutte A.J., van der Walt D.J., Gaylard M.J., MacLeod G.C. 1993, MNRAS, 1993, 261, 783

Caswell J.L., Vaile R.A., Ellingsen S.P., Whiteoak J.B., Norris R.P. 1995, MNRAS 1995, 272, 96

van der Walt D.J., Gaylard M.J., MacLeod G.C. 1995, A\&AS, 110, 81

Walsh A.J., Hayland A.R., Robinson G., Burton M.G. 1997, MNRAS, 291, 261

Slysh V.I., Val'tts I.E., Kalenskii S.V., Voronkov, M.A., Palagi F., Tofani G., Catarzi M. 1999, A\&AS, 134, 115

Szymczak M., Hrynek G., Kus, A.J. 2000, A\&AS, 134, 269

Minier V., Conway J.E., \& Booth R.S. 2001, A\&A, 369, 278 